\title{
Structure of phase III of solid hydrogen
}

\author{
CHRIS J. PICKARD ${ }^{1 *}$ AND RICHARD J. NEEDS² \\ ${ }^{1}$ Scottish Universities Physics Alliance, School of Physics and Astronomy, University of St Andrews, St Andrews, Fife KY16 9SS, UK \\ ${ }^{2}$ Theory of Condensed Matter Group, Cavendish Laboratory, Cambridge CB3 OHE, UK \\ *e-mail: cjp10@st-andrews.ac.uk
}

Hydrogen, being the first element in the periodic table, has the simplest electronic structure of any atom, and the hydrogen molecule contains the simplest covalent chemical bond. Nevertheless, the phase diagram of hydrogen is poorly understood. Determining the stable structures of solid hydrogen is a tremendous experimental challenge ${ }^{1-3}$, because hydrogen atoms scatter X-rays only weakly, leading to low-resolution diffraction patterns. Theoretical studies encounter major difficulties owing to the small energy differences between structures and the importance of the zero-point motion of the protons. We have systematically investigated the zerotemperature phase diagram of solid hydrogen using firstprinciples density functional theory (DFT) electronic-structure methods ${ }^{4}$, including the proton zero-point motion at the harmonic level. Our study leads to a radical revision of the DFT phase diagram of hydrogen up to nearly $400 \mathrm{GPa}$. That the most stable phases remain insulating to very high pressures eliminates a major discrepancy between theor $y^{5}$ and experiment ${ }^{6}$. One of our new phases is calculated to be stable over a wide range of pressures, and its vibrational properties agree with the available experimental data for phase III.

The low-pressure phase I of solid hydrogen, which consists of freely rotating molecules on a hexagonal close-packed lattice ${ }^{2}$, transforms at pressures of about $110 \mathrm{GPa}$ to the broken-symmetry phase II, in which the mean molecular orientations are ordered, and then to phase III at about $150 \mathrm{GPa}$ (ref. 1). However, even the combination of X-ray and neutron scattering data and Raman and infrared vibrational data has not so far yielded the structures of phases II and III of hydrogen.

The theoretical prediction of stable crystal structures is very difficult because of the need to search the very large space of possible structures, and the necessity of obtaining accurate energies for each of these structures. First-principles DFT methods have proved an efficient means of calculating quite accurate energies, and they have provided many insights into the properties of materials, including solid hydrogen ${ }^{5,7}$. At present, DFT offers the highest level of theoretical description at which we can carry out searches over many possible candidate structures.

Our approach is to relax many random structures to minima in the enthalpy at fixed pressure ${ }^{8}$. This method does not rely on previous theoretical or experimental results, and it allows for the possibility of finding radically new structures. In some cases we used the intuition gained from the random searches to build other candidate structures. We then calculated the enthalpies of the most stable phases at a larger number of pressures, generating the data shown in Fig. 1. We refer to each structure by its short HermannMauguin space-group symbol, giving additional information where an ambiguity might occur.
The lowest-enthalpy structures found around $100 \mathrm{GPa}$ were those of space groups $P c a 2_{1}$ and $P 2_{1} / c$, which were considered in previous studies, ${ }^{5,7}$, and a new structure of space group $P 6_{3} / \mathrm{m}$, which is marginally the most stable up to $105 \mathrm{GPa}$. The centres of the molecules in these structures form a slightly distorted hexagonal close-packed lattice, as is generally thought to occur in phase II of solid hydrogen. The molecular bond lengths of the different structures are very similar and the molecules are orientated so that the atoms of neighbouring molecules are kept apart. The $P 6_{3} / m$ structure differs from the other two in that some molecules lie perpendicular to the plane, whereas the others are arranged around it in a 'swirl'; see Fig. 2.

The most stable phase we found in the range 105-270 GPa is a layered structure of space group $C 2 / c$, which is illustrated in Fig. 3. We also found a layered molecular structure of Cmca symmetry with a 12-atom primitive unit cell. We refer to this as Cmca-12 to distinguish it from the Cmca structure discussed in earlier work $^{5}$, which was also generated by our searches. Cmca-12 is illustrated in Fig. 4, and it is the most stable phase in the range 270-385 GPa. From 385-490 GPa we found the Cmca structure to be the most stable, and above $490 \mathrm{GPa}$ the monatomic $I 4_{1} /$ amd structure with a $c / a$ ratio greater than unity (which is also the structure of Cs-IV) is most stable. Cmca-12 remains competitive up to about $500 \mathrm{GPa}$, and a more closely packed version of $\mathrm{Cmca}$, which we refer to as high-Cmca (and is the same structure as black phosphorus), is almost degenerate with $\mathrm{Cmca}-12$ and $\mathrm{I} 4_{1} /$ amd at about $480 \mathrm{GPa}$. A structure of $\mathrm{Cmcm}$ symmetry has been obtained by following an unstable phonon displacement of the $P c a 2_{1}$ structure $^{9}$. We have studied this structure but found it to be uncompetitive; see Fig. 1. We found numerous less stable structures, including a particularly intriguing family of 'mixed' structures consisting of alternate layers of strongly bonded hydrogen molecules and less strongly bonded atoms, which at higher pressures resemble graphene layers. These mixed structures are described in more detail in the Supplementary Information.

The small mass of the proton poses a significant problem for theoretical descriptions of hydrogen; the zero-point ( $\mathrm{ZP}$ ) motion of the nuclei is large enough to significantly affect the relative stabilities of structures and their vibrational properties. We have estimated ZP vibrational energies within DFT using the harmonic approximation. The harmonic approximation is likely to give useful estimates of the total $\mathrm{ZP}$ vibrational energy of the candidate structures at low pressures, as the calculated harmonic vibronic frequencies are similar to the measured values. There are, however, noticeable anharmonic effects in hydrogen, even at low pressures ${ }^{1,10}$. In monatomic structures, which are believed to be stable at very high pressures, the harmonic approximation fails completely ${ }^{11}$. 


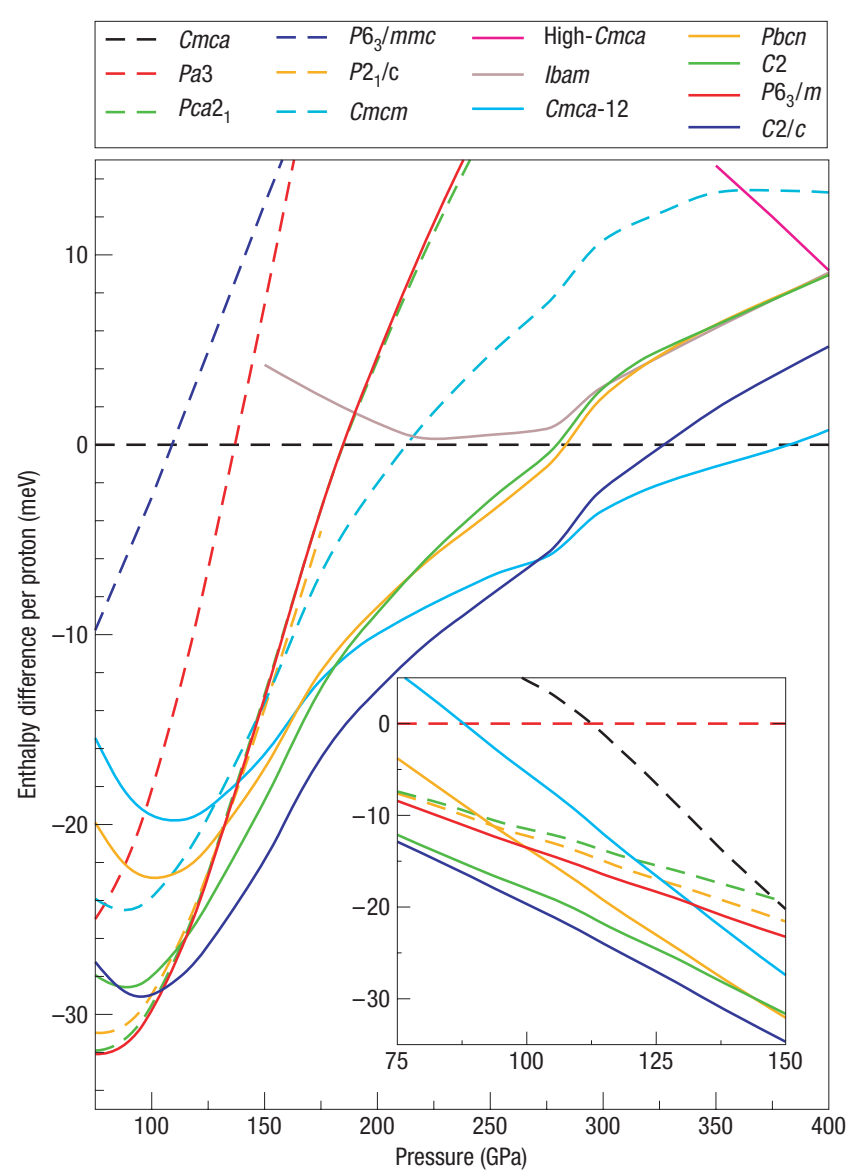

Figure 1 Enthalpy per proton as a function of pressure. Static lattice enthalpies relative to the Cmca phase. Inset: Enthalpies including ZP motion, relative to the Pa3 phase. The solid lines indicate our new structures and the dashed lines those considered previously.

Our calculated ZP energy of the free hydrogen molecule of $0.133 \mathrm{eV}$ per proton is a little larger than the experimental value of $0.129 \mathrm{eV}$ per proton ${ }^{12}$, the majority of the error arising from the harmonic approximation. The calculated ZP energies at $75 \mathrm{GPa}$ are roughly $0.25 \mathrm{eV}$ per proton, rising to about $0.28 \mathrm{eV}$ per proton at $150 \mathrm{GPa}$. The ZP pressures are quite small, being roughly $6 \mathrm{GPa}$ at a static lattice pressure of $75 \mathrm{GPa}$, rising to roughly $8 \mathrm{GPa}$ at $150 \mathrm{GPa}$. The ZP enthalpies are large on the scale of Fig. 1. There is a strong tendency for them to cancel between the different phases, but the resulting changes in their relative stabilities are significant. Comparing the main and inset graphs of Fig. 1, we see that ZP effects favour the $C 2 / c$ and $C m c a$ phases and the mixed $C 2$ and $P b c n$ phases over the $P 2_{1} / c, P c a 2_{1}$ and $P 6_{3} / m$ phases. The predicted range of stability of $C 2 / c$ then extends to lower pressures. The ZP effects reduce the upper pressure at which $C 2 / c$ is stable to $240 \mathrm{GPa}$, where it yields to Cmca-12, although $\mathrm{Pbcn}$ is very close in enthalpy at this pressure.

Our results lead to a complete revision of the DFT phase diagram of hydrogen. Within the static-lattice approximation, the most stable phases are $P 6_{3} / m$ ( $\left.<105 \mathrm{GPa}\right), C 2 / c$ (105-270 GPa), Cmca-12 (270-385 GPa) and Cmca (385-490 GPa), followed by $I 4_{1} / a m d$. Of these phases, only Cmca and $I 4_{1} / a m d$ were known previously. When ZP effects are included, $\mathrm{C} 2 / c$ becomes stable below $240 \mathrm{GPa}$ and Cmca-12 above. The enthalpies of some of the phases are very similar, and small changes in their relative

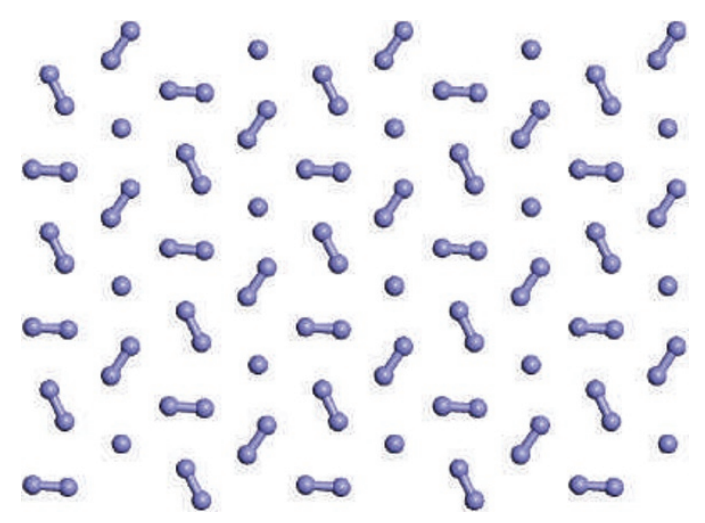

Figure $2 \mathrm{~A}$ layer of the hexagonal $\boldsymbol{P G}_{3} / \boldsymbol{m}$ structure at $\mathbf{3 0 0} \mathbf{G P a}$. The layers are stacked in an $A B A B$ fashion, and the primitive unit cell contains 16 atoms, which form two types of hydrogen molecule. Three-quarters of the molecules lie flat within the plane, and one-quarter lie perpendicular to the plane. The centres of the molecules lie on a slightly distorted hexagonal close-packed lattice. In this and Figs 3 and 4 , bonds are indicated between atoms closer than $1.05 \AA$, and close contacts (pink dashed lines), if they exist, between atoms closer than $1.15 \AA$.

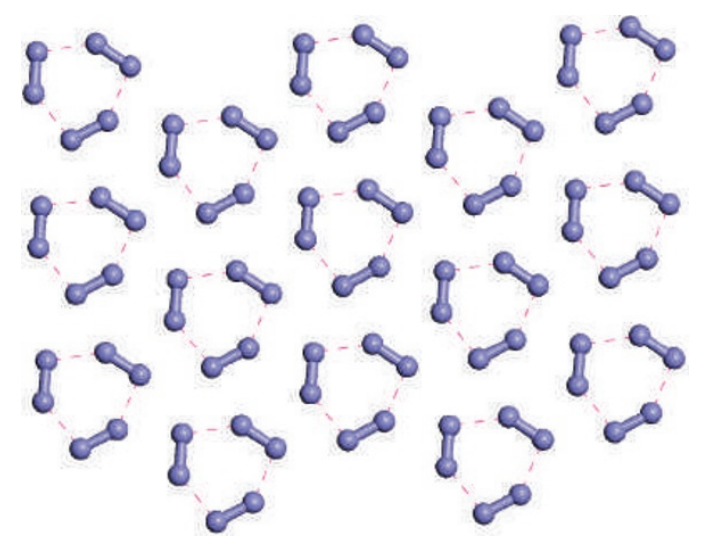

Figure 3 A layer of the monoclinic $\boldsymbol{C 2} / \boldsymbol{c}$ structure at $300 \mathrm{GPa}$. The layers are arranged in an ABCDA fashion, and the primitive unit cell contains 24 atoms. Each layer consists of three inequivalent molecules whose axes are nearly parallel to the plane and whose centres form a distorted hexagonal lattice. Note the asymmetry between the two ends of the molecules, which leads to the strong infrared activity in this structure.

enthalpies could significantly alter the stability regions of the different phases.

The calculated densities of electronic states show that $\mathrm{Cmca}-12$ becomes metallic at about $240 \mathrm{GPa}, \mathrm{C} 2$ at about $260 \mathrm{GPa}$ and the $\mathrm{C} 2 / \mathrm{c}$ and $\mathrm{Pbcn}$ phases at about $300 \mathrm{GPa}$. Given that standard DFT approaches normally underestimate bandgaps, the true metallization pressures are likely to be significantly higher. Exact-exchange DFT calculations indicate that the true bandgaps in hydrogen solids are roughly $1-2 \mathrm{eV}$ higher than the standard DFT values $^{13}$. Increasing our calculated gaps by $1 \mathrm{eV}$ and extrapolating to the pressure at which the gap vanishes gives metallization pressures of about $350 \mathrm{GPa}$ for $\mathrm{Pbcn}$ and $\mathrm{C} 2 / \mathrm{c}$, whereas an increase of $2 \mathrm{eV}$ gives metallization at about $410 \mathrm{GPa}$. These pressures are consistent with the experimental finding that hydrogen remains insulating up to at least $320 \mathrm{GPa}$ (ref. 6). 


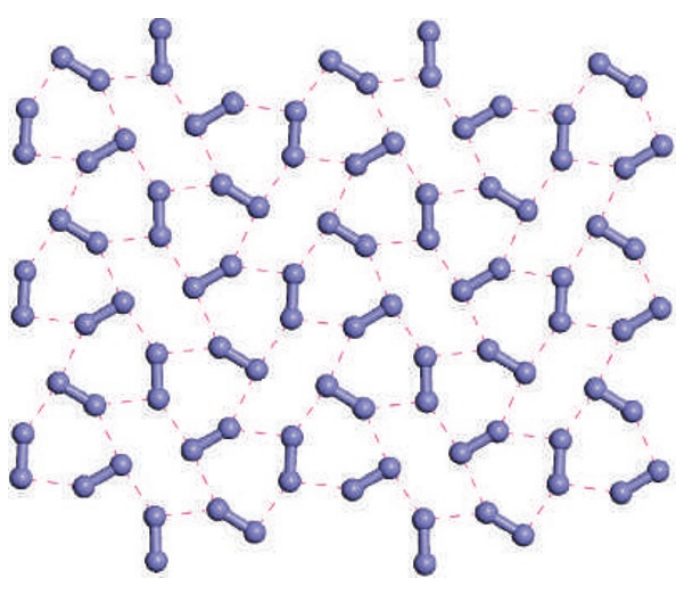

Figure 4 A layer of the monoclinic $\mathrm{Cmca-12}$ structure at $300 \mathrm{GPa}$. The layers are arranged in an $\mathrm{ABA}$ fashion, and the primitive unit cell contains 12 atoms. The arrangement of the molecules is similar to that in $C 2 / C$, although the molecules lie flat within the layer and the distortion from hexagonal packing is larger.

We have calculated the infrared intensities of the vibrational modes and identified the Raman-active vibron modes of the most significant structures; see Fig. 5 and the Supplementary Information. Phase II seems to have three infrared-active vibron modes, but overall its infrared activity is $\operatorname{low}^{1} . P c a 2_{1}$ has three infrared-active vibron modes and a low overall infrared activity, whereas $P 6_{3} / \mathrm{m}$ has only one infrared-active vibron and a significantly larger overall infrared activity. $P 6_{3} / m$ is therefore inconsistent with the infrared data.

The structures formed by the heavier isotope deuterium are expected to be similar to those of hydrogen. Recent experiments ${ }^{3}$ indicate that phase II of deuterium has an incommensurate structure, although a periodic model of $P \overline{3} m 1$ symmetry was suggested as having the same local ordering of molecules. We found this molecular structure to be poorly packed, and relaxation with the constraint that the symmetry is not lowered led to a structure of higher $\mathrm{PG}_{3} / m m c$ symmetry, which, however, is high in enthalpy, as shown in Fig. 1. We therefore conclude that the even less stable $P \overline{3} m 1$ structure is unlikely to be related to phase II. (A similar conclusion was arrived at in ref. 14.) On the other hand, the neutron data for deuterium of ref. 3 were found to be incompatible with the $P c a 2_{1}$ and $P 2_{1} / c$ structures.

The very small volume change and apparently continuous behaviour of the Raman vibron frequency across the I-II transition suggests that phase II may consist of an arrangement of molecules on a hexagonal, or nearly hexagonal, close-packed lattice. Although the $P 2_{1} / c, P c a 2_{1}$ and $P 6_{3} / m$ structures are of this type, it is possible that none of the structures suggested so far is compatible with phase II of hydrogen or deuterium, and the level of theory used may be inadequate. For example, our calculations do not account for the differences between ortho and para molecules, which plays an important role in phases I and II of hydrogen and deuterium ${ }^{15}$. Although the mean molecular orientations are ordered in phase II, very wide-angle librational motion occurs, and the phase coherence between the orientations of molecules is weak. Our DFT calculations show that a number of packings of molecules (all on the basis of a slightly distorted hexagonal close-packed lattice) have very similar enthalpies in the pressure range appropriate for phase II, suggesting that several significantly different local molecular configurations might contribute to the

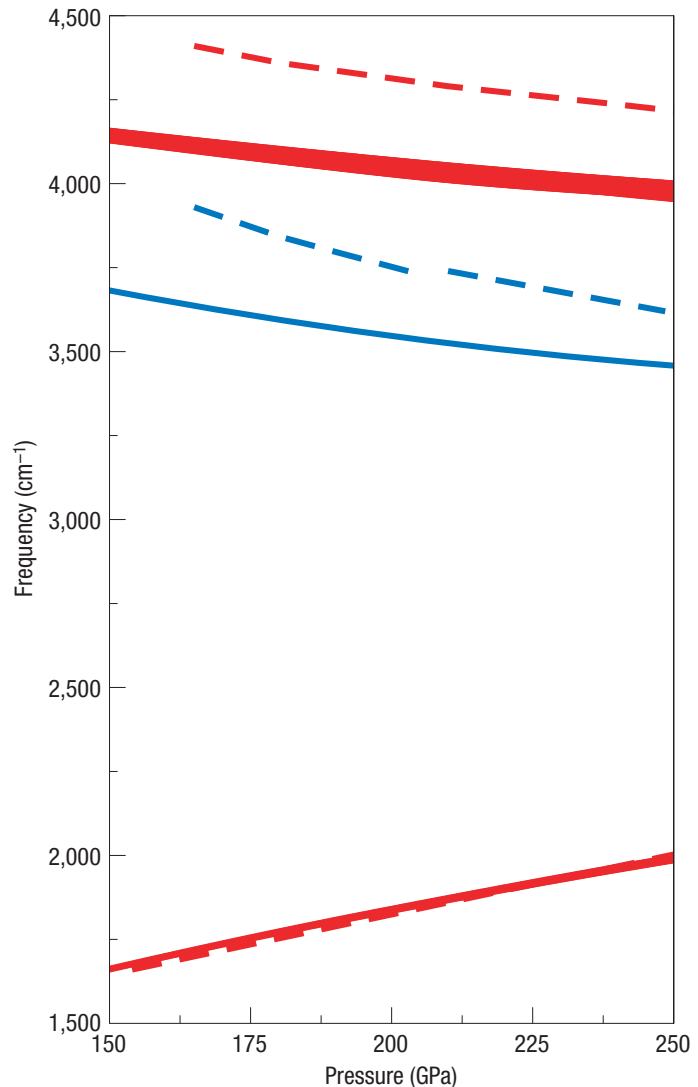

Figure 5 Vibrational frequencies of the $C 2 / c$ structure compared with experimental data for phase III. Only the most strongly infrared-active modes are shown; the intensities of all other infrared-active modes are at least an order of magnitude smaller. The calculated infrared frequencies are shown as red solid lines, with the line width being proportional to the intensity. The Raman-active vibron mode is shown as a blue solid line. Experimental data from ref. 19 are shown as dashed lines.

overall structure. A full understanding of phase II presents a difficult theoretical challenge.

The distinction between ortho and para molecules is believed to be unimportant in phase III because the molecules have much less freedom to rotate in this compressed structure. Two of the most notable characteristics of phase III are its much greater infrared activity than phase II and the appearance of a single intense infrared active vibron peak ${ }^{16}$. The Cmca-12 structure shows two fairly weakly infrared-active vibron modes separated by between 200 and $250 \mathrm{~cm}^{-1}$. The overall infrared intensity of $\mathrm{Cmca}-12$ is calculated to be just over twice that in $P c a 2_{1}$ and is actually less than that in $\mathrm{P6}_{3} / \mathrm{m}$, and it seems likely that the infrared intensity of Cmca-12 is too small to be consistent with phase III. The Cmca phase, found to be stable over a very large pressure range in previous DFT studies ${ }^{5}$, is ruled out as a candidate for phase III because it is metallic and its vibrons are much softer than the observed ones, and we have found considerably more stable phases over most of the relevant pressure range.

Our new $C 2 / c$ structure is a strong candidate for phase III. $C 2 / c$ shows intense infrared vibron activity arising from the increased charge asymmetry between the two ends of the hydrogen molecules; see Fig. 3. Our calculations show an increase in overall infrared activity in $\mathrm{C} 2 / \mathrm{c}$ over that in $P c a 2_{1}$ by a factor of six. The increase for the vibron modes is much larger, the infrared 
intensity of the vibrons in $C 2 / c$ being about 150 times larger than in $P c a 2_{1} . C 2 / c$ has six infrared-active vibrons, three of which are strong and differ by less than $25 \mathrm{~cm}^{-1}$ over a very wide range of pressures (see Fig. 5) and would therefore appear as a single peak in the infrared absorption spectrum, in agreement with experiment. The two strongest differ by less than $15 \mathrm{~cm}^{-1}$, and account for $95 \%$ of the intensity of the combined peak. The intensities of the other three infrared-active vibrons are each less than $1 \%$ of the intensity of the strong infrared vibron peak. The infrared activity of the strong infrared-active vibrons in $C 2 / c$ increases with pressure, as is observed in phase $\mathrm{III}^{17}$. Figure 5 shows the variation with pressure of both the strong infrared peak and the Raman-active vibron frequency of $C 2 / c$ to be in good agreement with experiment ${ }^{18}$. Figure 5 also shows a fairly strongly infraredactive phonon mode between 1,600 and $2,000 \mathrm{~cm}^{-1}$, as seen in experiment ${ }^{18}$. The Supplementary Information shows that the calculated libron frequencies (those up to about $600 \mathrm{~cm}^{-1}$ ) appear in the correct frequency range and gently increase with pressure in a similar fashion to those observed in experiment ${ }^{18}$.

\section{METHODS}

We used the CASTEP plane-wave code ${ }^{19}$, the Perdew-Burke-Ernzerhof generalized gradient approximation density functiona ${ }^{20}$ and ultrasoft pseudopotentials ${ }^{21}$. We carried out very extensive random searches at pressures from 100 to $600 \mathrm{GPa}$ in steps of $100 \mathrm{GPa}$, using unit cells containing eight and 12 atoms, and other searches using up to 48 -atom cells at some pressures. We calculated the phonon frequencies for the ZP energies using a finite-difference method and the infrared intensities using density functional perturbation theory ${ }^{22}$; see the Supplementary Information for more details.

Received 1 December 2006; accepted 30 April 2007; published 27 May 2007.

\section{References}

1. Mao, H. K. \& Hemley, R. J. Ultrahigh-pressure transitions in solid hydrogen. Rev. Mod. Phys. 66 671-692 (1994)

2. Loubeyre, P. et al. X-ray diffraction and equation of state of hydrogen at megabar pressures. Nature 383, 702-704 (1996).
3. Goncharenko, I. \& Loubeyre, P. Neutron and X-ray diffraction study of the broken symmetry phase transition in solid deuterium. Nature 435, 1206-1209 (2005).

4. Martin, R. M. Electronic Structure: Basic Theory and Practical Methods (Cambridge Univ. Press, Cambridge, 2004).

5. Johnson, K. A. \& Ashcroft, N. W. Structure and bandgap closure in dense hydrogen. Nature $\mathbf{4 0 3}$ $632-635(2000)$

6. Loubeyre, P., Occelli, F. \& LeToullec, R. Optical studies of solid hydrogen to $320 \mathrm{GPa}$ and evidence for black hydrogen. Nature 416, 613-617 (2002).

7. Kohanoff, J., Scandolo, S., Chiarotti, G. L. \& Tosatti, E. Solid molecular hydrogen: The broken symmetry phase. Phys. Rev. Lett. 78, 2783-2786 (1997).

8. Pickard, C. J. \& Needs, R. J. High pressure phases of silane. Phys. Rev. Lett. 97, 045504 (2006).

9. Kohanoff, J., Scandolo, S., de Gironcoli, S. \& Tosatti, E. Dipole-quadrupole interactions and the nature of Phase III of compressed hydrogen. Phys. Rev. Lett. 83, 4097 (1999).

10. Ashcroft, N. W. Pairing instabilities in dense hydrogen. Phys. Rev. B 41, 10963-10971 (1990).

11. Natoli, V., Martin, R. M. \& Ceperley, D. M. Crystal structure of atomic hydrogen. Phys. Rev. Lett. 70, 1952-1955 (1993).

12. Dabrowski, I. The Lyman and Werner bands of $\mathrm{H}_{2}$. Can. J. Phys. 62, 1639-1664 (1984).

13. Städele, M. \& Martin, R. M. Metallization of molecular hydrogen: Predictions from exact-exchange calculations. Phys. Rev. Lett. 84, 6070-6073 (2000).

14. Zhang, L. J. et al. Ab initio lattice dynamics evidence for the broken-symmetry phase of solid hydrogen. J. Phys. Condens. Matter 18, 9917-9927 (2006).

15. Goncharov, A. F., Eggert, J. H., Mazin, I. I., Hemley, R. J. \& Mao, H. K. Raman excitations and orientational ordering in deuterium at high pressure. Phys. Rev. B 54, 15590-15593 (1996).

16. Hemley, R. J., Soos, Z. G., Hanfland, M. \& Mao, H.-K. Charge-transfer states in dense hydrogen. Nature 369, 384-387 (1994).

17. Hemley, R. J., Mazin, I. I., Goncharov, A. F. \& Mao, H. K. Vibron effective charges in dense hydrogen. Europhys. Lett. 37, 403-407 (1997).

18. Goncharov, A. F., Gregoryanz, E., Hemley, R. J. \& Mao, H.-K. Spectroscopic studies of the vibrational and electronic properties of solid hydrogen to $285 \mathrm{GPa}$. Proc. Natl Acad. Sci. USA $\mathbf{9 8}$, 14234-14237 (2001).

19. Clark, S. J. et al. First principles methods using CASTEP. Z. Kristallogr. 220, 567-570 (2005).

20. Perdew, J. P., Burke, K. \& Ernzerhof, M. Generalized gradient approximation made simple. Phys. Rev. Lett. 77, 3865-3868 (1996)

21. Vanderbilt, D. Soft self-consistent pseudopotentials in a generalized eigenvalue formalism. Phys. Rev. B 41, 7892-7895 (1990)

22. Refson, K., Tulip, P. R. \& Clark, S. J. Variational density functional perturbation theory for dielectrics and lattice dynamics. Phys. Rev. B 73, 155114 (2006).

\section{Acknowledgements}

C.J.P. was supported by an EPSRC Advanced Research Fellowship. We thank K. Refson for assistance in carrying out the phonon calculations and J. Kohanoff for useful discussions, including the information that he and G. Grad had also studied the Cmca-12 phase.

Correspondence and requests for materials should be addressed to C.J.P.

Supplementary Information accompanies this paper on www.nature.com/naturephysics.

\section{Competing financial interests}

The authors declare competing financial interests: details accompany the full-text HTML version of the paper at www.nature.com/naturephysics/.

Reprints and permission information is available online at http://npg.nature.com/reprintsandpermissions/ 